\title{
Fatigue corrosion crack extension across the interface of an elastic bi-material
}

\author{
A. P. Jivkov ${ }^{*}$ \\ Malmö University, Division of Solid Mechanics, SE-205 06 Malmö, Sweden
}

\begin{abstract}
In this work crack propagation in a bi-material composed of a thin elastic layer ideally bonded to a large elastic substrate is studied. A flaw is assumed existing on the surface of the layer. Cracks nucleate from the flaw and propagate through the bi-material. The cracks have realistic geometrical shapes, where the crack tip is an integral part of the crack surface. Thus the crack propagation is associated with a crack surface evolution. Material loss due to corrosion of the crack surface is the physical ground for the evolution. A controlling mechanism for the surface advancement is the rupture of a brittle corrosionprotective film, which is continually building-up along the corroding surface. The rate of surface evolution is a function of the degree of protective film damage, caused by the surface straining. This leads to a moving boundary formulation, for which a numerical solution is proposed. Fatigue loading is considered as a suitable way to maintain crack evolution at a constant peak load level. Under the assumed model, the cracks always pass the interface. The elastic mismatch is shown to influence the growth rate variation around the interface. Crack extensions are presented as functions of the elastic mismatch and as functions of the initial flaw size. It is shown how the results can be used in designing bi-material systems. A typical morphology evolution of a crack passing through an interface with a weak-stiff transition is presented. An example of fatigue corrosion fracture is offered, which shows that the crack morphology of the model resembles the one observed in reality. It is concluded that the realistic crack geometry is an effective concept and the moving boundary formulation could be a very successful tool for simulating realistic crack propagation.
\end{abstract}

Keywords: Bi-material structure; Crack evolution; Moving boundary; Fatigue corrosion; Finite elements.

\footnotetext{
*E-mail: andrey.jivkov@ts.mah.se, Fax: +46 (0)40 6657135
} 


\section{Nomenclature}

$K_{I} \quad$ stress intensity factor

$E, v \quad$ Young's modulus and Poisson's ratio of a homogeneous material

$H \quad$ realistic crack half-width

$\kappa \quad$ dimensionless load parameter

$X_{1}, X_{2} \quad$ Chartesian coordinates describing the body undeformed state

$W, D \quad$ surface flaw width and depth, respectively

$B \quad$ characteristic body dimension

$L \quad$ surface layer thickness

$\Lambda \quad$ dimensionless layer thickness: $\Lambda=L / W$

$x_{1}, x_{2} \quad$ Chartesian coordinates describing the body deformed state

$F_{i j} \quad$ deformation gradient components

$J$ deformation gradient Jacobian

$E_{1}, E_{2} \quad$ Young's moduli of the layer and the substrate, respectively

$M \quad$ elastic mismatch coefficient: $M=\left(E_{2}-E_{1}\right) /\left(E_{2}+E_{1}\right)$

$\alpha, \beta \quad$ Dundurs parameters

$\sigma_{i j} \quad$ Cauchy stress tensor components

$\varepsilon_{f} \quad$ rupture strain of the surface passive film

$\varepsilon_{s} \quad$ surface strain measure / film damage measure

$v_{s} \quad$ surface evolution rate

$v \quad$ electrochemical dissolution rate

$t$ physical time

$t_{0} \quad$ characteristic time in the corrosion law

$v_{0} \quad$ characteristic dissolution rate in the corrosion law

$p \quad$ exponent in the corrosion law

$U_{1}, U_{2}$ displacement vector components

$T_{1}, T_{2}$ boundary traction vector components

$u, u_{0} \quad$ cycle value and peak value of applied displacements magnitude, respectively

$T, T_{0} \quad$ period and peak duration of a rectangular cycle, respectively

$d_{s} \quad$ surface point advancement per load cycle

$d \quad$ electrochemical dissolution advancement per load cycle

$\delta \quad$ crack growth rate: $\delta=d_{s} / d$, taken at the crack tip

a crack length

$N \quad$ number of cycles

$t_{h} \quad$ crack arrival time at a given distance $L$ in a homogeneous material

$\delta_{h} \quad$ crack growth rate at a given distance $L$ in a homogeneous material

$a_{h} \quad$ crack length at a given time $t$ in a homogeneous material

\section{Introduction}

Fatigue and corrosion fatigue cracking are deterioration processes that often initiate from flaws existing on a material body surface. An improved mechanical and chemical performance of structural components is achieved by introduction of a protective surface layer. For instance, the layer could be a hard coating, intended to increase the wear resistance of a particular component. Or it could be a film, manufactured by various methods to improve the corrosion resistance. The choice of the material 
composition in such a bi-material system is crucial for the reliability of the structure, since it may lead to an accelerated fatigue crack growth. The problem of a crack advancing through a bi-material interface is therefore of fundamental importance in understanding the fracture behaviour of layered systems. This problem was firstly treated by Zak and Williams [1] for the case of ideally bonded linear elastic materials. It was shown there, that when the crack tip is placed exactly at the interface, the stress field surrounding the tip has a singularity exponent, which is no longer equal to one-half as in homogeneous materials. The exponent was found to depend on the elastic mismatch with values between zero and one. For exponent values different from onehalf the classical Griffith-Irwin fracture criterion cannot be directly applied, because the local stress intensity factor either grows infinitely or vanishes when the crack approaches the interface.

The problem for a crack advancing in an elastic bi-material solid was further investigated by Cook and Erdogan [2], Atkinson [3], Lu and Erdogan [4], He and Hutchinson [5] and Romeo and Ballarini [6]. All these works use integral equation methods to study various aspects of the problem - deducing expressions for the near-tip stress field and studying deflection versus penetration behaviour of the crack approaching the interface. However, the non-square-root stress singularity when the tip is exactly at the interface remains as an anomalous consequence of the accepted models for the crack and the bi-material system. To remedy that, a crack propagation criterion associated with non-linear processes may be suggested. For example Romeo and Ballarini [7] and Wäppling et al. [8] used a cohesive zone model with a propagation criterion based on a crack opening displacement. Another suggestion may be to abandon the ideal interface model and treat the zone between the two materials as a thin transition layer, where the properties change gradually from one material to the other. This was proposed and investigated by Atkinson [9] and Erdogan et al. [10]. Yet a third possibility is to equip the crack with a realistic geometrical shape, where the tip is not represented by a singular point but is an integral part of the smooth crack surface. This offers a very attractive layout, although introducing considerable mathematical complexity, for studying crack propagation, because the crack advance is identified with a smooth surface evolution. The realistic geometry could be idealised, under certain conditions, as a crack with a sharp tip. Such an approach requires a formulation of a moving boundary value problem, where the physically essential part is the constitutive law that governs the rate of surface evolution. A solution to the moving boundary problem, would provide answers not only to questions of qualitative character, as whether a crack is attracted or retarded by a bi-material interface, but also to some quantitative questions, as what the degree of interface influence is. The analytical complexity, however, that the moving boundary value problem introduces, demands a trade-off. To follow extensive crack propagation, a numerical solution to the problem is imperative.

In this work, the propagation of a realistic crack across a bi-material interface is studied. The bi-material system is composed of a thin elastic surface layer, ideally bonded to a substrate of a different elastic material. The crack nucleates from a surface flaw and propagates through the interface under fatigue corrosion conditions. Existence of a brittle passive film along the corroding surface is presupposed. The evolution of the crack surface is a result of the material dissolution, which takes place in the areas of passive film damage. The interaction between the surface deformation and the brittle film determines whether the film is intact or ruptured, i.e. it controls the accessibility of 
bare solid to the corrosive environment. The material loss per unit time and unit surface area, which corresponds to the rate of surface evolution, is assumed proportional to the degree of the passive film damage. The proportionality factor is physically interpreted as the dissolution rate, which may be determined from electrochemical considerations. Thus, a simple constitutive law relates the evolution rate with the deformed state of the surface. The geometrical and the physical description of the model, as well as a short note on the numerical technique used, are presented in section 2. The same physical settings with a different geometry and numerical scheme were used by Jivkov and Ståhle [11] to study the influence of the realistic crack geometry on the stress corrosion crack growth rate. It was shown there, that the role of the crack shape in terms of shallowness and sharpness was small compared to the role of the crack width. The crack growth rate was found to be a function of the dimensionless load parameter

$$
\kappa=\frac{K_{I}}{E \sqrt{H}},
$$

where $K_{I}$ is the far-field stress intensity factor, $E$ is the material elastic modulus, $H$ is the crack half-width, and all local linear measures scale with $H$. For $\kappa<0.1$, i.e. strains at the tip less than $10 \%$, the growth rate was proportional to the load parameter. This observation is used in section 3.2, where the results for a crack propagating in a homogeneous material are presented. Section 3.3 is dedicated to cracks growing in bimaterial systems. The influence of the elastic mismatch and the initial flaw size on the crack evolution is studied. A real-life example, presented in section 4, provides a positive support, at least qualitatively, to the results obtained with the proposed moving boundary formulation for realistic crack propagation.

\section{Model and method}

A plane bi-material body is considered. The body consists of an elastic substrate and a thin layer of a different elastic material. The surface of the layer is assumed in contact with a corrosive environment. An elliptically shaped flaw of width $W$ and depth $D$ is supposed existing on the corroding surface. The depth of the flaw is fixed to $D=0.1 \mathrm{~W}$. A Cartesian coordinate system $\left(X_{1}, X_{2}\right)$ is introduced with origin placed at the centre of the flaw. Figure 1 illustrates the geometry of the entire body (a) and the near-flaw region (b). The body occupies the rectangle $0 \leq X_{1} \leq B,-B \leq X_{2} \leq B$, where $B=10^{4} W$ is chosen. The layer has thickness $L$, expressed as a multiple of the flaw width as $L=\Lambda \mathrm{W}$. The shape of the flaw is described using a cubic B-spline curve, as is the evolving surface during the entire growth.

The body is considered to be in plane strain. Finite strain formulation of the body kinematics is utilised. If a material point with coordinates $\left(X_{1}, X_{2}\right)$ in the undeformed state of the body, obtains coordinates $\left(x_{1}, x_{2}\right)$ after the deformation, the deformation gradient is defined as a tensor with components

$$
F_{i j}=\frac{\partial x_{i}}{\partial X_{j}}, \quad i, j=1,2 .
$$

The Jacobian of the deformation gradient is given by $J=\operatorname{det}\left[F_{i j}\right]$.

The materials of the layer and of the substrate have elastic moduli, $E_{1}$ and $E_{2}$, respectively and the same Poisson's ratio, $v=1 / 3$. The elastic mismatch coefficient is defined as $M=\left(E_{2}-E_{1}\right) /\left(E_{2}+E_{1}\right)$. Since finite strain formulation for the problem kinematics is used in the work, the results cannot, to the author's knowledge, be 
expressed in terms of Dunders parameters, $\alpha$ and $\beta$, (see e.g. [12]). However, if the strains involved during the studied crack propagation are small enough, so that the small strain approximation could be accepted, the Dunders parameters could be used to describe the elastic mismatch. In such cases, the two materials may have different elastic moduli, as well as different Poisson's ratios. In all results, the elastic mismatch $M$ is identified as $\alpha$, provided that $\beta=\alpha / 2$ is fulfilled. This requirement comes from the definition of the Dunders parameters and the considered plane strain conditions.

The constitutive relationship for both materials is of neo-Hookean type. The components of the Cauchy stress tensor at a point are given through the relation

$$
\sigma_{i j}=\frac{E_{m}}{J(1+v)}\left[\frac{1}{2}\left(F_{i j} F_{j i}-\delta_{i j}\right)+\frac{v}{(1-2 v)}(\ln J) \delta_{i j}\right], \quad i, j=1,2,
$$

where $m=1$ or 2 , depending on whether the point belongs to the material with elastic modulus $E_{1}$ or $E_{2}$, respectively. The body is in equilibrium with the externally applied loads when for every interior point of the body the following equations are fulfilled

$$
\frac{\partial \sigma_{i j}}{\partial x_{i}}=0, \quad i, j=1,2 \text {. }
$$

The corroding surface is assumed covered by a passive film, which being built of metal oxides is supposed brittle in tension. The film thickness does not enter the model. A single parameter - the strain at rupture, $\varepsilon_{f}$ - describes the film behaviour in tension. The rupture strain used in this work is $\varepsilon_{f}=0.001$, which is a physically reasonable value suggested in [13]. In the unloaded state of the body, the film is everywhere compact and prevents any dissolution of metal ions. Upon mechanical loading, the film deforms elastically together with the bulk material, which may be either the layer or the substrate material. If sufficiently large, the deformation leads to film rupture in certain regions where the corrosion process dissolves material and advances the surface in the normal direction. A detailed description of the surface deformation-film rupture process may be found in [11]. As a measure for surface strain is chosen the relative elongation of a differential surface arc element, defined as $\varepsilon_{s}=(d s-d S) / d s$, where $d S$ and $d s$ denote the arc lengths before and after deformation, respectively. The film ruptures at a point if $\varepsilon_{s}>\varepsilon_{f}$, and the film fragments between rupture points are assumed to relax. As a smeared measure for exposure of bare metal to the corrosive environment is taken the degree of film damage, defined as $\varepsilon_{s} \theta\left(\varepsilon_{s}-\varepsilon_{f}\right)$, where $\theta(x)$ is the Heaviside step function, i.e. $\theta(x)=1$ if $x>0$ and $\theta(x)=0$ otherwise. The suggested constitutive law relating the rate of surface advance, $v_{s}$, with the degree of film damage at a point reads

$$
v_{s}=v \varepsilon_{s} \theta\left(\varepsilon_{s}-\varepsilon_{f}\right)
$$

where $v$ is the dissolution rate, a parameter generally depending on the solid-solution composition and varying in time. Note that $\varepsilon_{s}<1$, which limits the rate of surface advance to the dissolution rate for a bare metal. Although the physical reasoning given above for the linear relation (5) is not experimentally justified, it is thought as a good first approximation of a surface advance law.

Following Engelhardt et al. [13] the dissolution rate is presented as

$$
v=\left\{\begin{array}{ll}
v_{0}, & t \leq t_{0} \\
v_{0}\left(\frac{t_{0}}{t}\right)^{p}, & t>t_{0}
\end{array},\right.
$$


where $t$ is the time measured from the exposure of bare surface, and $v_{0}, t_{0}$ and $p$ are electrochemical parameters, depending on the solid-solution composition and reflecting the dissolution and repassivation kinetics and the transport processes in the environment. The exponent in (6) is physically limited in the interval $0<p<1$. Hence, $v_{0}$ provides the maximum possible dissolution rate that may be observed under pure bare surface state.

The components of the displacement and the traction vectors with respect to the coordinate system $\left(X_{1}, X_{2}\right)$ are denoted by $U_{1}$ and $U_{2}$, and $T_{1}$ and $T_{2}$, respectively. The load is given through prescribed displacements along the two boundaries $0<X_{1}<B$; $\left|X_{2}\right|=B$. The displacements are applied in cycles, where for simplicity each cycle is assumed to have a rectangular shape with displacement magnitude

$$
u_{0}=\varepsilon_{f} B
$$

peak duration $T_{0}$, and $T>T_{0}$ is used to denote the period between two cycles. The boundary conditions over one cycle, $0<t<T$, are given by

$$
\begin{aligned}
& T_{1}=T_{2}=0, \quad \text { at } \quad X_{1}=0, \quad\left|X_{2}\right| \leq B . \\
& T_{2}=0 \quad \text { and } \quad U_{1}=0, \text { at } \quad X_{1}=B, \quad\left|X_{2}\right| \leq B \\
& T_{1}=0 \quad \text { and } \quad U_{2}=-u_{0}\left[\theta(t)-\theta\left(t-T_{0}\right)\right], \quad \text { at } \quad X_{2}=-B, \quad 0<X_{1}<B \\
& T_{1}=0 \quad \text { and } \quad U_{2}=u_{0}\left[\theta(t)-\theta\left(t-T_{0}\right)\right], \quad \text { at } \quad X_{2}=B, \quad 0<X_{1}<B
\end{aligned}
$$

Note that the traction free boundary at $X_{1}=0$ is the layer surface exposed to corrosion. The existing flaw is also traction free, as well as the evolving surface. The choice of $u_{0}$ ensures a constant strain field in a homogeneous body of the same geometry as in Figure 1, but without a flaw on the surface. The strain along the surface, $X_{1}=0$, will then be equal to the rupture strain of the passive film. Any small deviation from the flat surface will create sufficient strain concentration, so that the film ruptures and the dissolution process starts.

The advancement of the corroding boundary per load cycle, $d_{s}$, is obtained from (5) and (6) upon time integration over the cycle. It is understood here, that the dissolution actually occurs only during the cycle peak, $T_{0}$, whereas in the rest of the cycle period, ( $T$ $-T_{0}$ ), the film is compacted and the dissolution is prohibited. To write the integral of (6) in a compact form, introduce the function $\lceil x\rceil$, such that $\lceil x\rceil=x$ when $x<1$ and $\lceil x\rceil=1$ otherwise. The advancement of a surface point in the direction normal to the surface at that point then reads

$$
d_{s}=d \varepsilon_{s} \theta\left(\varepsilon_{s}-\varepsilon_{f}\right)
$$

where

$$
d=v_{0} T_{0}\left(\frac{1}{1-p}\left\lceil\frac{t_{0}}{T_{0}}\right\rceil^{p}-\frac{p}{1-p}\left\lceil\frac{t_{0}}{T_{0}}\right\rceil\right) .
$$

In summary, the model stated by (1)-(4) and (7)-(10) constitutes a discrete moving boundary value problem. Each load cycle is understood as a new boundary value problem. The body geometry for the new problem is determined from the entire past history of the evolving boundary. The equilibrium solution for the new problem defines the present evolution. A numerical solution is proposed for the moving boundary value problem. An in-house finite element program is employed to solve the boundary value problem. The surface strains, $\varepsilon_{s}$, provide the advance of the corroding surface via (9). The current body geometry is changed by displacing the corroding surface to its evolved position. Thus, the boundary value problem for the next cycle is prepared. In the finite 
element environment, the surface advance is represented by the surface nodes displacements. In order to properly follow the surface shape changes, a new distribution of nodes along the evolved surface is essential. A cubic B-spline curve through the displaced positions of the old nodes describes the evolved surface. The new nodes are introduced along the spline using curvature and spacing control parameters. For computational efficiency only the process region, i.e. the surface region with $\varepsilon_{s}>\varepsilon_{f}$, is maintained with a mesh fine enough to accurately describe the shape. The parts of the crack surface left behind the process region are described with a coarser mesh. This may lead to a "fading memory effect", where possible arrested crack branches are smoothed out after certain evolution of the main crack. The changed body geometry requires remeshing of the interior, which is completed with a Delauney-type triangulation procedure, following Schewchuk [14].

\section{Results}

\subsection{Homogeneous case}

The fatigue corrosion crack growth in a homogeneous material is firstly presented. Figure 2 shows the crack configuration after certain number of load cycles. The current crack length and crack half-width are denoted by $a$ and $H$, respectively. The crack length is measured from the boundary $X_{1}=0$ to the surface point with largest $X_{1}$ coordinate, which is called crack tip. The crack width, $2 H$, is defined as the distance between the points, where the two lines through the crack tip, forming $45^{\circ}$ with the crack plane, cross the crack faces. In the initial stages of evolution, a strain and dissolution localisation is observed, which leads to a crack nucleating from the pit. This transition period is omitted in the following, where the result is shown for $a>W$, i.e. $a$ $>10 D$. If an ideal crack of length $a$, emerging from a surface pit of width $W$ and depth $D$ is considered, the stress intensity factor determining the state of the near-tip region is given by (see e.g. [15])

$$
K_{I}=\sigma \sqrt{\pi a} F\left(\frac{a}{D}, \frac{W}{D}\right),
$$

where $\sigma$ is the stress applied at a large distance and perpendicular to the crack plane, and $F$ is a correction factor accounting for the geometry. In the considered case of geometry and loading, the stress at large distance during the peak of the cycle is given by $\sigma=\varepsilon_{f} E$, where $E$ is the elastic modulus of the homogeneous material. For $a \gg D$, the correction factor takes the form $F=1.1215 / \sqrt{1-D / a}$ (see [15]). Hence, a dimensionless load factor corresponding to (1) is written as

$$
\kappa=1.1215 \varepsilon_{f} \sqrt{\frac{\pi}{1-D / a}} \sqrt{\frac{a}{H}} .
$$

Introduce the normalised crack tip advance per cycle (crack growth rate) as $\delta=d_{s} / d$, which as seen from (9) represents the current degree of film damage at the tip. In terms of number of cycles, $N$, the tip advance per cycle is written as $d_{s}=\mathrm{d} a / \mathrm{d} N$, hence

$$
\delta=\left.\frac{d_{s}}{d}\right|_{\text {tip }}=\frac{\mathrm{d} a}{\mathrm{~d} N} \frac{1}{d}
$$

Note that $d$ is a physically based maximum advance per cycle determined using (10) for given electrochemical parameters, $v_{0}, t_{0}$ and $p$, and peak duration of a cycle, $T_{0}$. In this 
framework, the physical time is given as $t=N T$, via the number of cycles, $N$, and the cycle period, $T$, hence the ratio between two time intervals is the same as the ratio between the corresponding number of cycles.

The crack growth rate, $\delta$, as a function of $\kappa$ determined via (12) is shown in Figure 3. The largest value of $\kappa$ corresponds to a crack length of about $50 \mathrm{~W}$. The approximately linear relationship between $\delta$ and $\kappa$, with a nearly unit proportionality factor coincides with the result for a long crack in an infinite elastic body, found valid for $\kappa<0.1$. This demonstrates the existence of a square-root singular stress field enclosing the growing realistic crack tip in a homogeneous material. The results for a crack in a homogeneous material are used for scaling the bi-material results via the following parameters: $a_{h}$ is the crack length at a given time $t ; t_{h}$ is the crack arrival time at a given distance $L ; \delta_{h}$ is the crack growth rate at a given distance $L$.

\subsection{Bi-material cases}

Numerical simulations have been performed for the bi-material model geometry, varying the layer thickness from $\Lambda=1$ to $\Lambda=10$, and the elastic mismatch in the interval $-9 / 11<M<9 / 11$. Note, that positive values of the mismatch represent weaker layer than substrate, while negative values represent weaker substrate than layer. As an illustration for a weak layer-stiff substrate composition, Figure 4 shows a typical crack configuration when the moving front is around the interface (a), and when the crack has grown a certain distance into the substrate (b). The particular values of the variable parameters are, $\Lambda=5$ and $M=0.5$, as also depicted in the figure.

The crack growth rate is plotted as a function of the crack extension in Figure 5, for $\Lambda=5$ and for two bi-material compositions - a weaker layer with $M=0.5$ (a) and a stiffer layer with $M=-0.5$ (b). In the figure, the crack extension, $a$, is scaled with the layer thickness, $L$, and the crack growth rate, $\delta$, is scaled with the growth rate of a crack in a homogeneous material, $\delta_{h}$, achieved on cracks arrival at a distance $5 W$. Additionally, the growth rate versus extension for a crack in a homogeneous material is shown in both plots with dashed lines. An interesting observation, depicted on the plots, is that the decrease or the increase of the crack growth rate fulfils the approximate relation $\delta / \delta_{h} \approx E_{1} / E_{2}$. Such a relation was found in all the studied cases: fixed elastic mismatch $|M|=0.5$ and variable layer thickness $\Lambda=1,2,3, \ldots, 10$; and fixed layer thickness $\Lambda=5$ and variable elastic mismatch $|M|=0.2,0.33,0.5, \ldots, 0.82$. The deviation from this approximate equality, found for the considered cases, may be written in the form $\left(E_{1} \delta_{h}\right) /\left(E_{2} \delta\right)=1 \pm 0.15$.

Figure 6 shows the elastic mismatch dependence of the crack arrival time at the interface (a), and of the crack extension, $a$, at three chosen time instances (b). The results for $\Lambda=5$ are used. In both plots, $t_{h}$ represents the time necessary for a crack in a homogeneous material to reach an extension $5 \mathrm{~W}$. In the plot (b), $a_{h}$ is the extension of a crack in a homogeneous material at time $t$. The three time instances on that plot are chosen in order to show the $M$-dependence of short cracks $\left(t=0.5 t_{h}, a<L\right)$, moderate cracks $\left(t=1.1 t_{h}, a \approx L\right)$, and long cracks $\left(t=2.5 t_{h}, a>L\right)$. The terms short, moderate and long designate only the crack length relative to the layer thickness.

The influence of the layer thickness, $\Lambda$, on the crack growth behaviour is presented in Figure 7, where the crack extensions, $a$, are given as functions of the time, $t$, for a weaker layer with $M=0.5$ (a) and for a stiffer layer with $M=-0.5$ (b). For clarity, the graphs are given only for three different values of the layer thickness, $\Lambda=1,5$ and 10 . 
In addition, the graph for a crack in a homogeneous material is plotted with a solid line in both (a) and (b). The crack extension is scaled with the initial flaw width, $W$, and the time scale, $t_{h}$, is the same as defined for Figure 6.

\section{Discussion}

The moving boundary value problem, formulated in section 2 , offers an advantageous method to study evolution of cracks with realistic geometries. The driving force for the evolution is the momentary equilibrium of the considered solid body. From a purely mechanical perspective this equilibrium may be thought unstable at every instance of time, because of the excess of boundary conditions prescribed on the moving boundary. On that boundary both essential, via equation (5), and natural, via equation (8a), conditions are specified. The instability compels the boundary to evolve, such that the redistributed stresses are maintained in an instantaneous equilibrium with the applied loads. Abstracting the physical background of the model - the controlling process of passive film formation - the solution of the moving boundary value problem would provide a continuous evolution of the moving boundary under constant applied loads. When the passive film build-up is accounted for, an active loading is an essential prerequisite to maintain a surface advancement. In this work the active loading is considered as fatigue loading, but that is not an essential requirement. It is believed, that qualitatively the same results would be achieved if the loads were considered increasing in steps of sufficient duration to let the repassivation process restore compactly the passive film. One inadequacy between the model and the electrochemical processes that operate during the stress corrosion cracking is the assumption of a constant dissolution rate in the model (5). In reality the dissolution rate would change with the crack advance, mainly because of the limitations posed by transport processes in the solution side. To account for that, it would be necessary to solve a complementary moving boundary problem formulated over the solution domain and with the mass conservation as a condition for equilibrium.

The problem of a realistic crack nucleating from a surface flaw and propagating in a homogeneous material was firstly studied in order to gain confidence in the numerical technique used to solve the moving boundary problem. As Figure 3 demonstrates, the technique provides an acceptable approximate solution for the crack evolution, because the growing crack tip is shown embedded in the expected $K_{I}$-controlled fields. Note, that the load parameter, $\kappa$, does not depend on the material characteristics under the assumed plane strain conditions. This makes $\kappa$ a desirable substitute for the stress intensity factor, $K_{I}$, when studying cracks of realistic geometries interfering with bi-material interfaces.

Figure 4 illustrates a typical crack morphology evolution in a weak layer-stiff substrate material system. In an initial evolution phase, the crack shape and growth rate follow closely those of a crack in a homogeneous material, as seen also in plot (a) of Figure 5. This initial phase extends approximately to half the layer thickness for the considered elastic mismatch and initial flaw size. After that the crack growth rate in the bi-material decreases compared to growth rate in the homogeneous material. There may be two different causes for the decrease, as observed in the evolution process. When the crack tip is still far from the interface, $0.5 L<$ a $<L$ in plot (a) of Figure 5, crack deflection and branching trigger a growth rate reduction. The largest drop in the growth rate occurs at the interface, because the arriving crack tip flattens down over the stiffer substrate. The growth rate, however, does not vanish at the interface, as is the 
theoretical prediction for an ideal crack in an ideally bonded bi-material. The explanation for that lays on the assumed model for surface evolution and on the instability of the free surface formed at the interface. The model given by (5) or (9) states that a surface point would evolve whenever $\varepsilon_{s}>\varepsilon_{f}$ is fulfilled at the point. Upon arrival at the interface the flattened crack front is not an ideally flat surface, which causes several cracks to emerge from the areas where $\varepsilon_{s}>\varepsilon_{f}$, see picture (a) of Figure 4. Eventually, one of these cracks continues propagating into the substrate, while the others arrest, see picture (b) of Figure 4.

As a qualitative support for the model behaviour, a real-life example of fatigue corrosion cracking in a bi-material system is shown in Figure 8. Photograph (a) presents the cross section of a pressure vessel component consisting of a cladding (in a lighter colour) and a substrate (in a darker colour). The cladding is manufactured of a stainless steel with $20 \% \mathrm{Cr}$ and $10 \% \mathrm{Ni}$ and has a better corrosion properties than the substrate, i.e. a lower corrosion rate. The substrate is a pressure vessel steel of type SA 533 C11, which is stiffer than the cladding. The stress corrosion crack initiated at the cladding surface and propagated towards the interface between the two materials. The crack changed growth direction several times. These path changes are not necessarily due to the presence of the interface, but perhaps due to the cladding microstructure. Photograph (b) shows the magnified region close to the interface, where the crack had passed into the pressure vessel steel. It is seen there, that a pit-like formation was shaped at the interface, before a number of cracks initiated and propagated into the substrate. All these cracks, except one, arrested in the substrate. The crack tip region of the eventually propagating crack is shown in photograph (c). Figure 8 clearly shows the realistic shape of the corrosion crack and the fact that the crack passes into the stiffer material. That happens in spite of the blunting at the interface that takes a form of a pit. There may be various factors of metallurgical, electrochemical and mechanical nature that lead to the observed behaviour. In this work for example, micro-structural properties are not accounted for and plasticity is not considered. The corrosion growth rate is assumed constant and equal for both materials. In addition the growth model is deterministic, hence the crack morphology features obtained are a consequence of the uncertainties in the numerical procedure, while in reality these features are due to material heterogeneity. Still, the proposed model and solution provide a satisfactory illustration of the crack morphology close to the bi-material interface.

The results for the arrival time and crack extension as functions of the elastic mismatch, presented in Figure 6, show an unexpected behaviour for the stiff layer-weak substrate system, i.e. $M<0$. The cracks in such a system are delayed in the initial stages of growth, where the delay becomes significant for very stiff, compared to substrates, layers. This would imply that for short, compared to the layer thickness, cracks it is preferable to manufacture as stiff as possible coating on the base material. However, for practically reasonable elastic mismatches in stiff-weak composition, as $0>M>-0.5$, the delay effect is insignificant, compared to the delay effect shown for the weak-stiff systems. In a long run it is always preferable to have a combination of a weaker layer and a stiffer substrate.

The influence of the layer thickness on the crack evolution can be estimated from the results shown in Figure 7. A coating design example will clarify one possible use of the curves given in plot (a). Let the choice of the two materials be such that the elastic mismatch coefficient is $M=0.5$, and let the width of the largest expected surface flaw be $W$. Further, assume that from a fatigue corrosion experiment performed with a 
homogeneous material the time of crack arrival at distance $5 W$, i.e. $t_{h}$, is known. Actually, any measurement of the crack length, $a$, at a given time, $t$, is sufficient to determine $t_{h}$, because of the proportionality between the time and the square-root of the crack extension, which is easily seen from Figure 3. Under such conditions one may ask what is the layer thickness that yields the longest possible period of operation before the crack reaches a certain length, where component reparation or replacement is necessary. The answer to this question is easily read from plot (a) of Figure 7. If the allowed crack extension before inspection was $7.5 \mathrm{~W}$, the best choice for a layer thickness would be $L$ $=5 \mathrm{~W}$, at least among the given three cases of $\Lambda$. The time gained by using such a bimaterial composition instead of a homogeneous material can be calculated and is denoted by $\Delta t_{7.5}$ in the figure. If the allowed crack extension was $15 \mathrm{~W}$, then among the given plots the best choice would be a layer with a thickness $L=10 \mathrm{~W}$, and the time gained with respect to a homogeneous material is denoted by $\Delta t_{15}$.

The crack delay that may be achieved in the initial stages of growth, $a<<$, through a stiff layer-weak substrate bi-material with $M=-0.5$ is shown in plot (b) of Figure 7. The important observation is that upon approach to the interface the crack is significantly accelerated with respect to a crack in a homogeneous material. Therefore, application of stiffer layers than substrates should be made with caution. It may be tempting in some situations to use very stiff layers with $M<-0.5$, in order to increase the crack delay as much as possible (see Figure 6). This may be applied if the allowed crack extensions before component replacement are small compared to the layer thickness. However, it may be a dangerous approach if the moment of inspection is missed and the crack is left to advance close to the interface. If the stiff-weak transition is a must, curves of the type shown in plot (b) may be used in the design of the layer thickness in the same way as for the weak-stiff transition (a).

\section{Conclusions}

The study of a crack propagating across a bi-material interface is facilitated by the concept of realistic crack geometry. This concept allows one to treat crack propagation as an evolution of a smooth crack surface. Thus, a fracture criterion is not needed. Instead a surface evolution law is engaged, which is offered by the corrosion phenomenon in a physically pure form. The study of a continuous surface evolution was done using a simple evolution law and a moving boundary value problem formulation. This is shown to be a very fruitful approach, although the proposed solution to the problem is purely numerical. The numerically obtained crack morphology is qualitatively very similar to the one observed in the real-life example. The model prediction is that, irrespective of the elastic mismatch, the cracks would always pass the interface. In some cases, that happened after crack deflection and branching, but the cracks were not arrested or infinitely accelerated as the classical crack model predicts. However, the elastic mismatch is found to determine the degree of growth rate decrease or increase at the interface.

This rate variation makes the crack in the bi-material delayed or outdistanced by the corresponding crack in a homogeneous material. It is proposed how the presented results for the crack extension variations as functions of the elastic mismatch and the surface flaw size are to be used in designing bi-material systems.

\section{Acknowledgements}


The financial support, coming from the Swedish Centre for Nuclear Technology is highly appreciated.

\section{References}

[1] Zak AR, Williams, ML. Crack point stress singularities at a bi-material interface. J Appl MechTrans ASME 1963; 30: 142-143.

[2] Cook TS, Erdogan F. Stresses in bonded materials with a crack perpendicular to the interface. Int J Eng Sci 1972; 10: 667-697.

[3] Atkinson C. On the stress intensity factors associated with cracks interacting with an interface between two elastic media. Int J Eng Sci 1975; 13: 489-504.

[4] Lu MC, Erdogan F. Stress intensity factors in two bonded elastic layers containing cracks perpendicular to and on the interface. Eng Fract Mech 1983; 18 (3): 491-506, 507-528.

[5] He MY, Hutchinson JW. Crack deflection at an interface between dissimilar elastic materials. Int J Solids Struct 1989; 25 (9): 1053-1067.

[6] Romeo A, Ballarini R. A crack very close to a bimaterial interface. J Appl Mech-Trans ASME 1995; 62: 614-619.

[7] Romeo A, Ballarini R. A cohesive zone model for cracks terminating at a bimaterial interface. Int J Solids Struct 1997; 34 (11): 1307-1326.

[8] Wäppling D, Gunnars J, Ståhle P. Crack growth across a strength mismatched bimaterial interface. Int J Fract 1998; 89: 223-243.

[9] Atkinson C. On stress singularities and interfaces in linear elastic fracture mechanics. Int J Fract 1977; 13 (6): 807-820.

[10] Erdogan F, Kaya AC, Joseph PF. The mode III crack problem in bonded materials with a nonhomogeneous interfacial zone. J Appl Mech-Trans ASME 1991; 58: 419-427.

[11] Jivkov AP, Ståhle P. Strain-driven corrosion crack growth - a pilot study of intergranular stress corrosion cracking. Eng Fract Mech 2002; 69 (18): 2095-2111.

[12] Dundurs J. Edge-Bonded Dissimilar Orthogonal Elastic Wedges Under Normal and Shear Loading. J Appl Mech-Trans ASME 1969; 36: 650-652.

[13] Engelhardt GR, Urquidi-Macdonald M, Macdonald DD. A simplified method for estimating corrosion cavity growth rates. Corros Sci 1997; 39 (3): 419-441.

[14] Shewchuk JR. Delaunay refinement algorithms for triangular mesh generation. Comput Geom Theory Appl 2002; 22: 21-74.

[15] Tada H, Paris PC, Irwin GR. The Stress Analysis of Cracks Handbook. New York: ASME Press; 2000. 


\section{Figure captions}

Figure 1. Schematic of the model geometry: a) entire body; b) near-flow region.

Figure 2. Evolved crack geometry in a homogeneous material after a number of load cycles.

Figure 3. Crack growth rate, $\delta$, versus load, $\kappa$, in a homogeneous material.

Figure 4. Evolved crack geometry in a bi-material with $\Lambda=5$ and $M=0.5$ : a) crack tip region at the interface; b) crack tip in the substrate.

Figure 5. Crack growth rate versus crack extension in a bi-material with $\Lambda=5$ : a) $M=$ 0.5 ; b) $M=-0.5$.

Figure 6. Interface arrival time (a), and crack extensions (b), as functions of the elastic mismatch, $M$, for $\Lambda=5$.

Figure 7. Crack extensions as functions of time for $\Lambda=1,5$ and 10: a) $M=0.5$; b) $M=$ -0.5 .

Figure 8. Stress corrosion crack in a bi-material pressure vessel component: a) cross section; b) magnification of the interface region; c) magnification of the crack tip region. Reproduced with the permission from Vattenfall, Sweden. 
Figure 1.

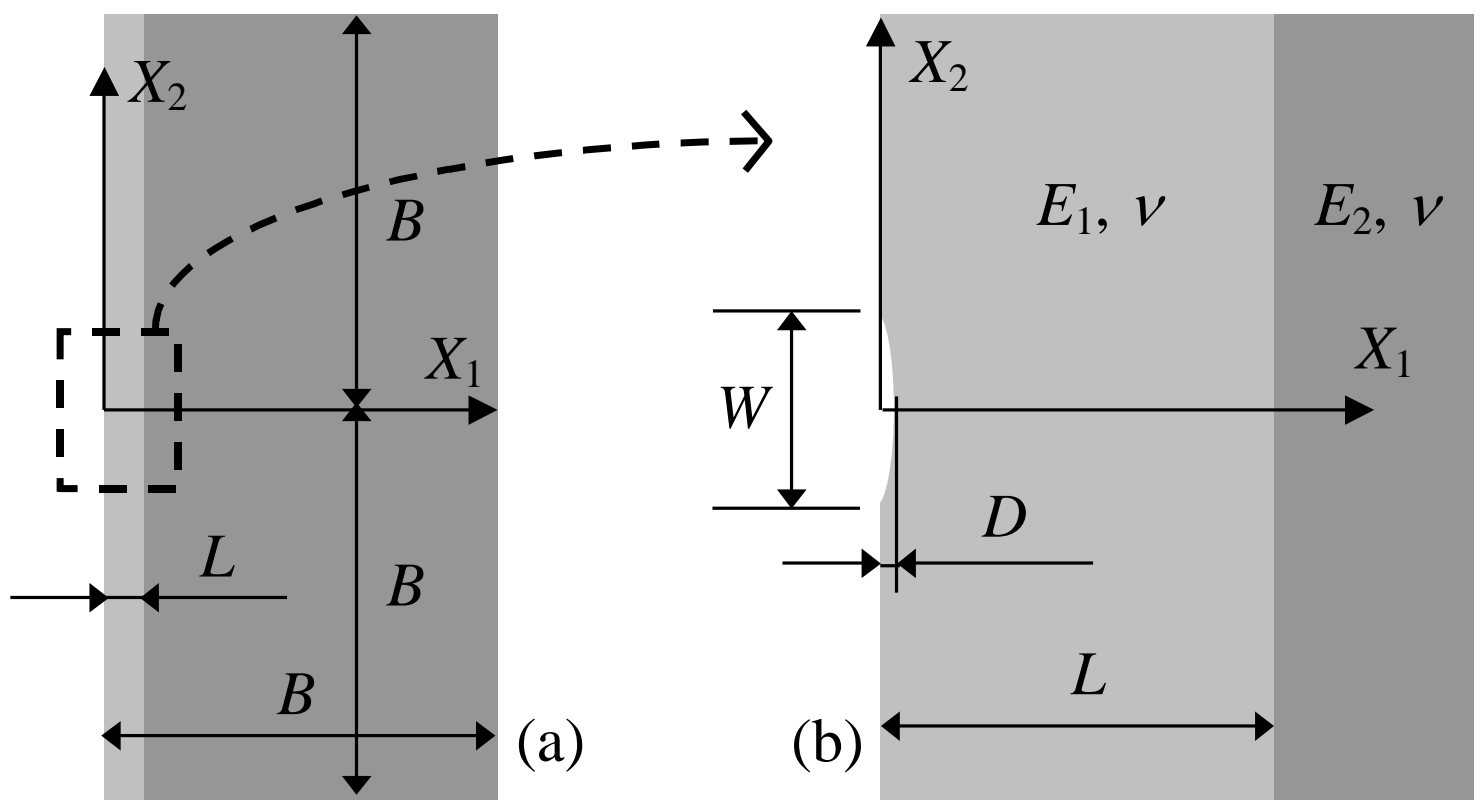


Figure 2.

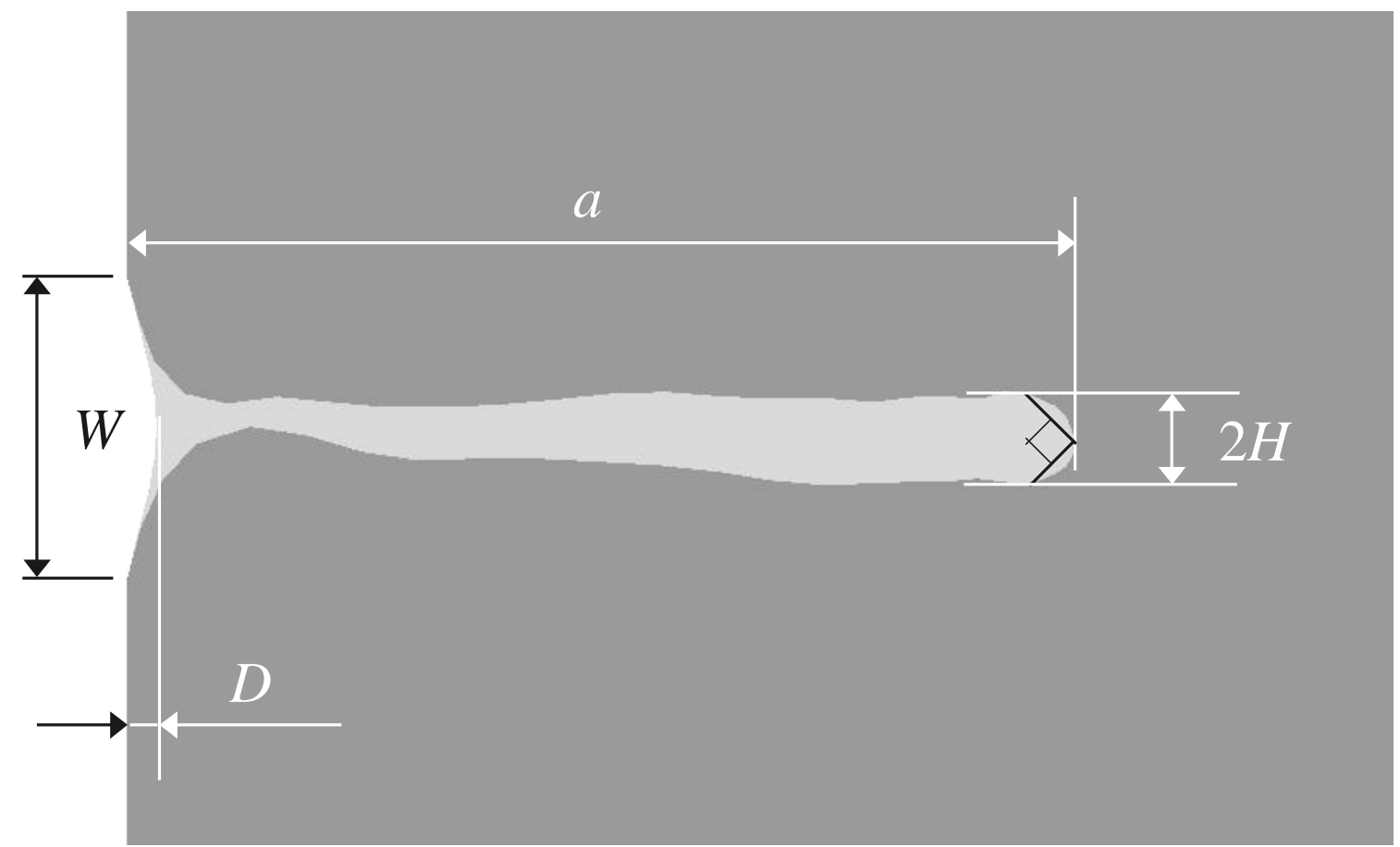


Figure 3.

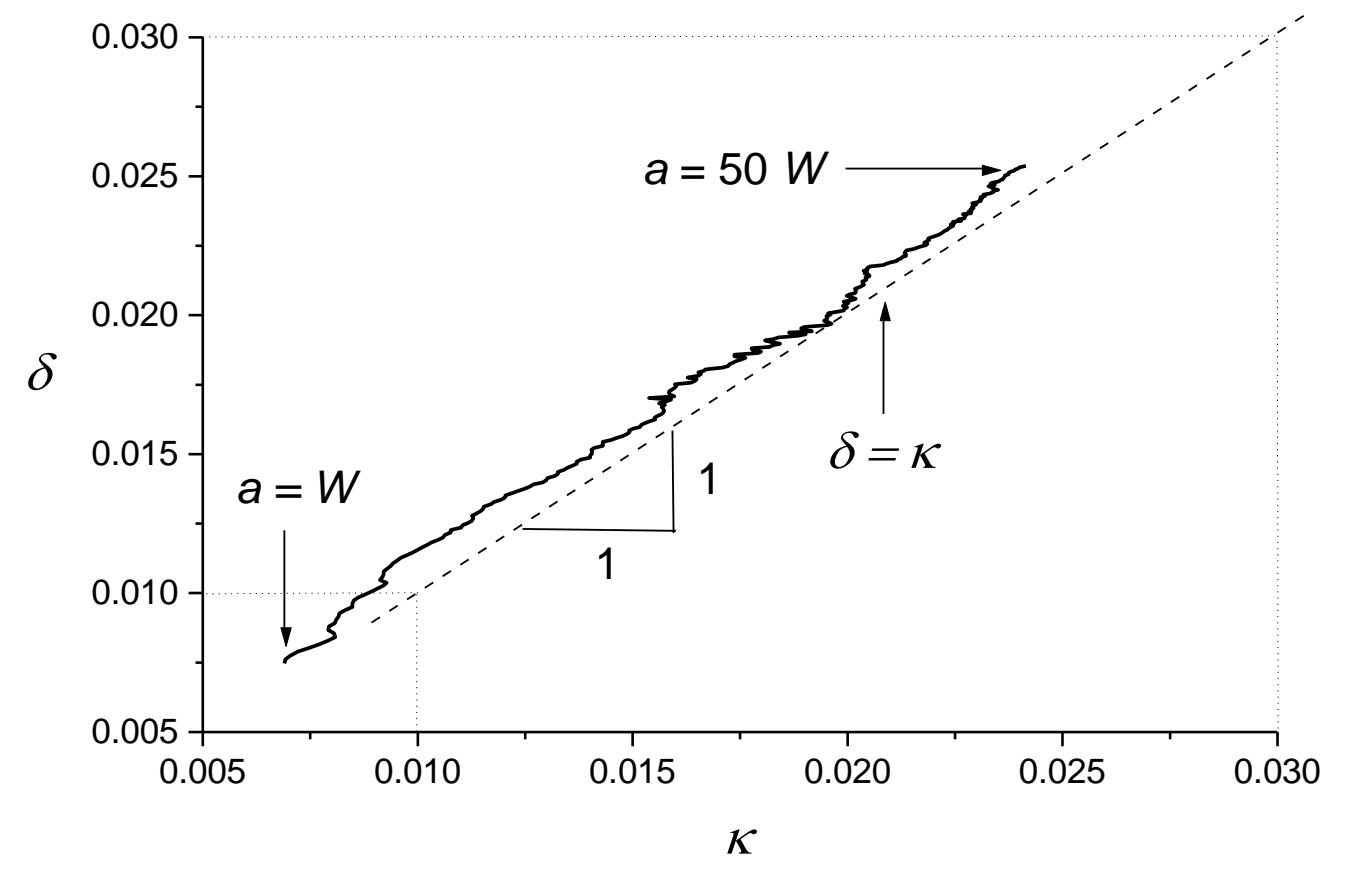


Figure 4.
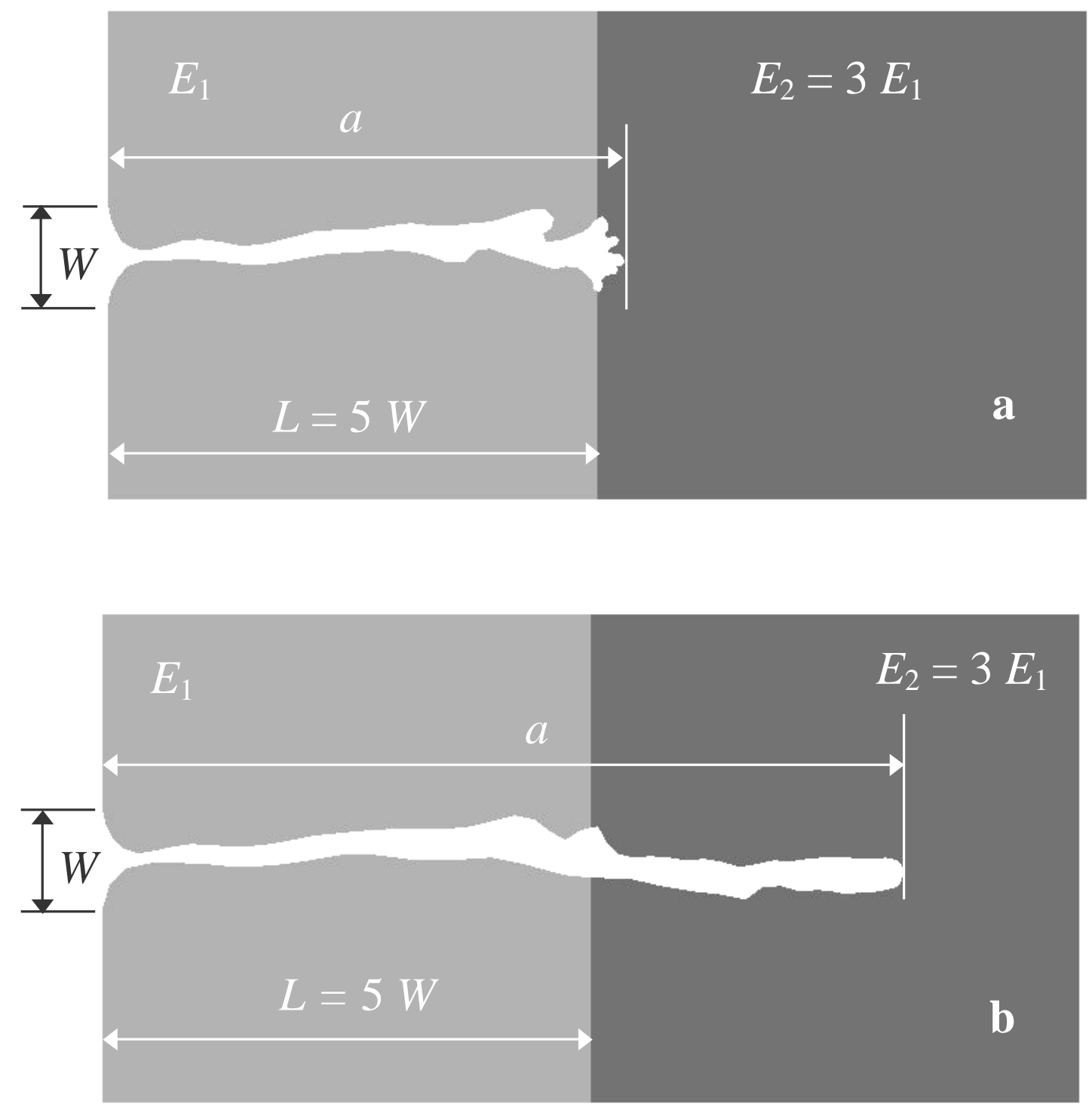
Figure 5.
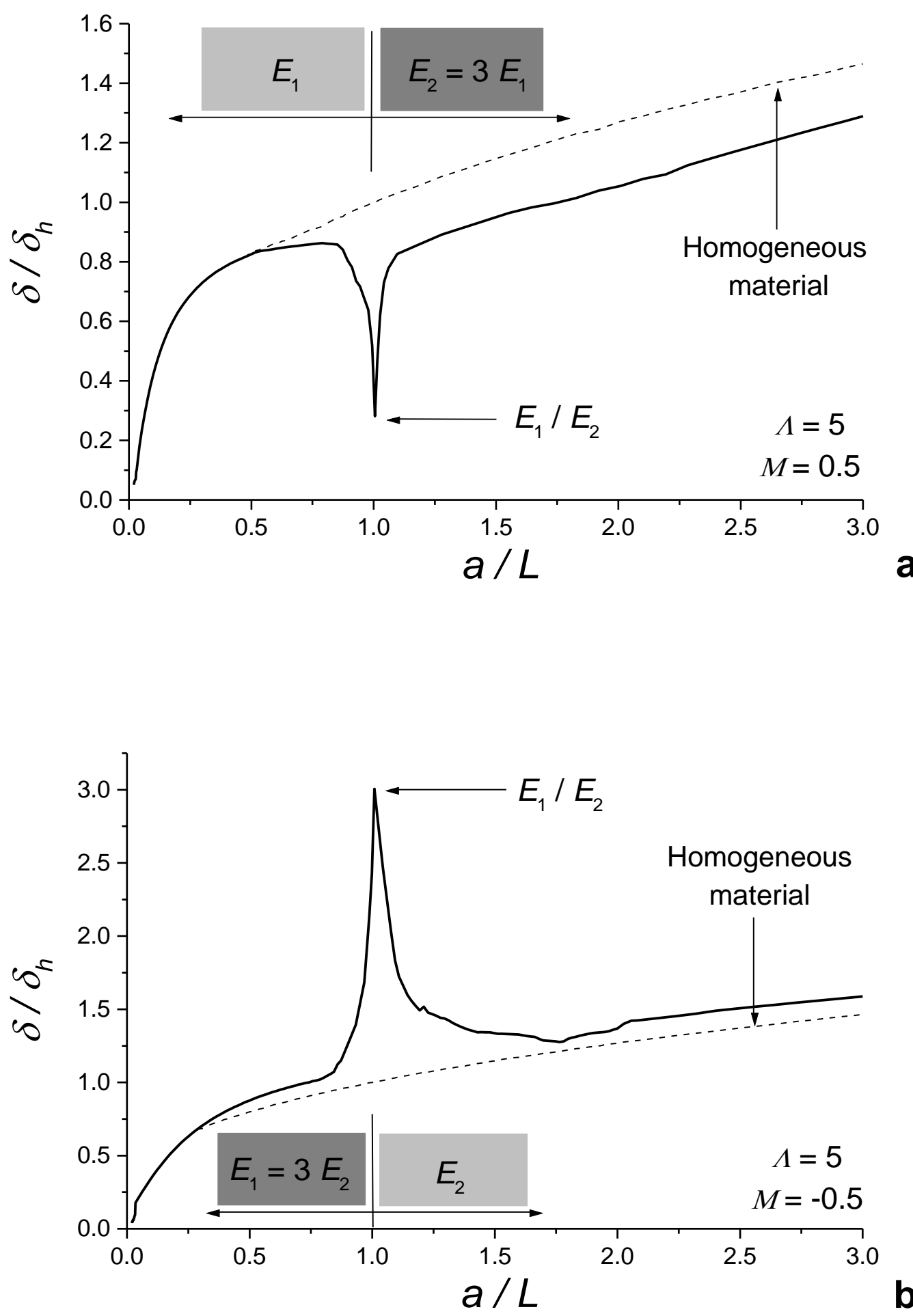
Figure 6.
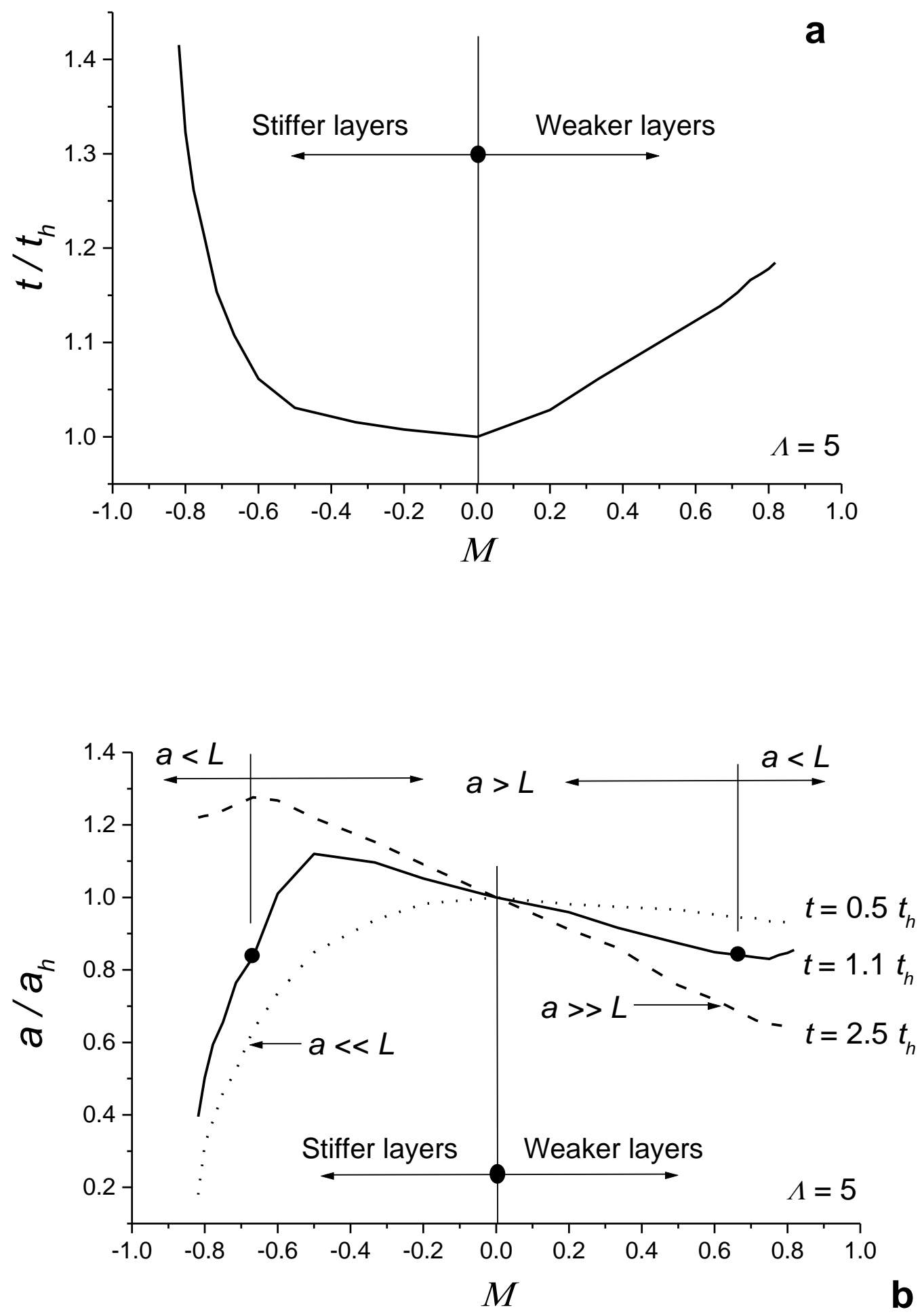
Figure 7.
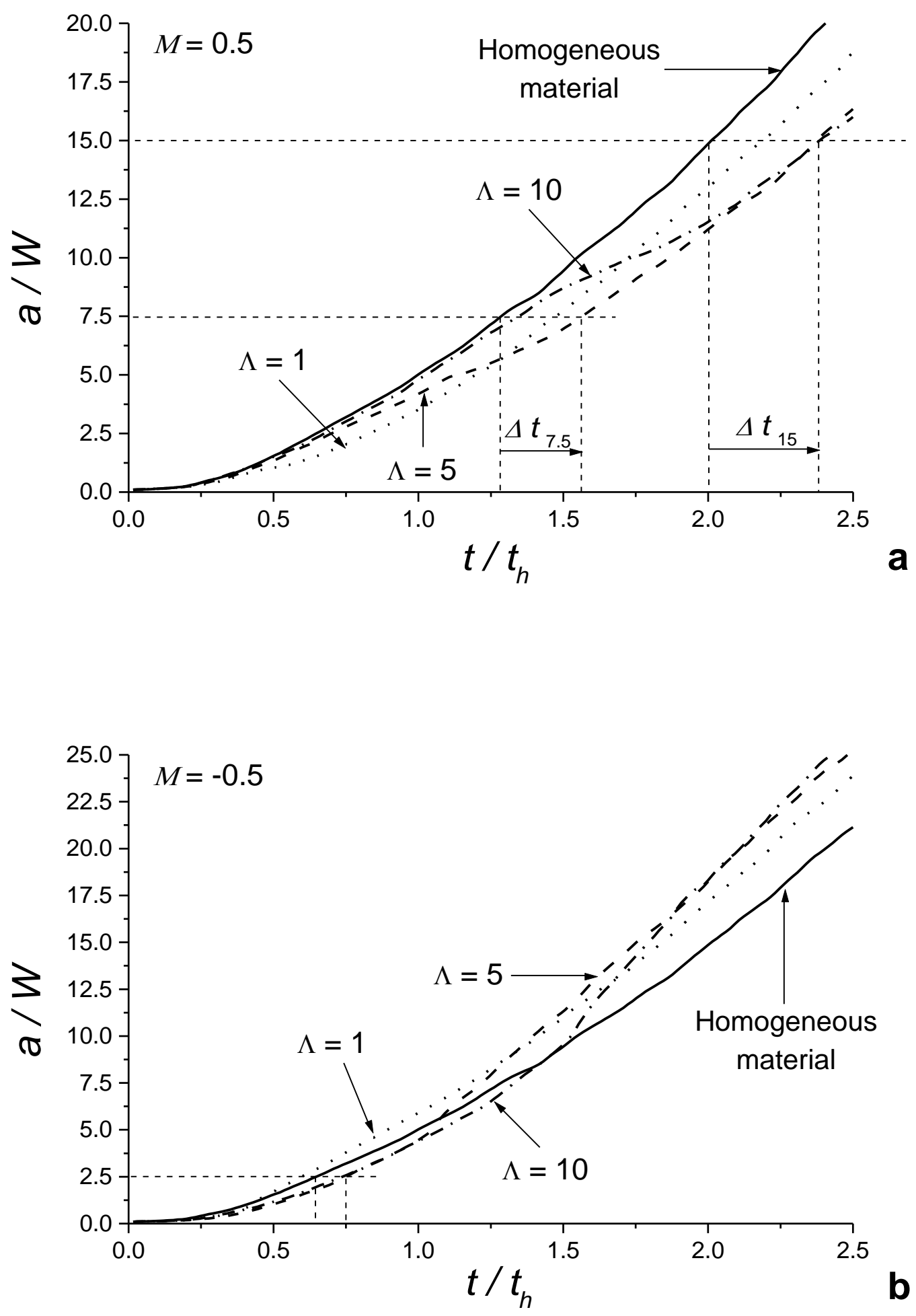
Figure 8.
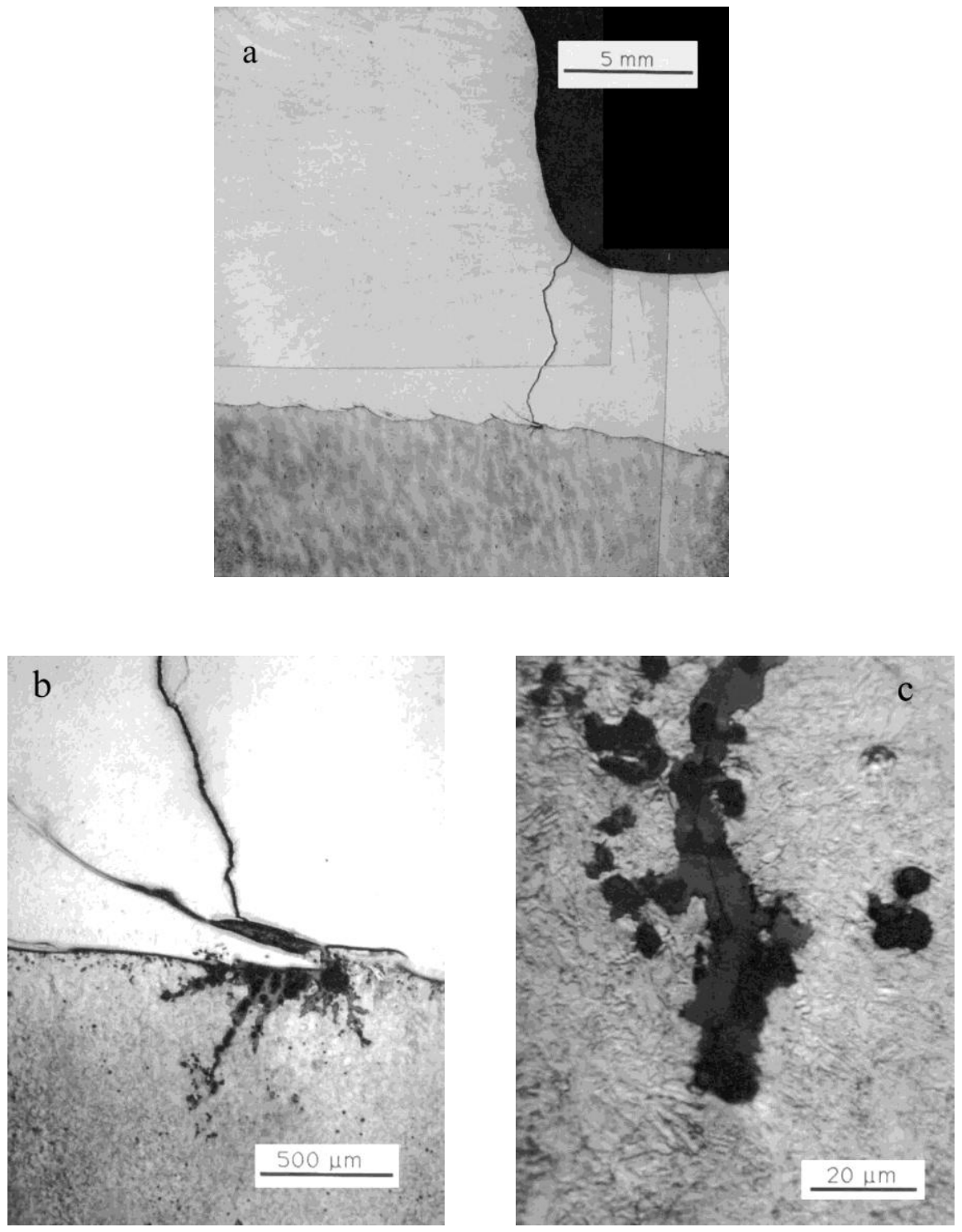